\title{
Polythiophene doping of metal-organic frameworks using innate MOF-catalyzed oxidative polymerization ${ }^{\dagger}$
}

\author{
Nicholas Marshall ${ }^{a *}$, William James ${ }^{a}$, Jeremy Fulmer ${ }^{a}$, Scott Crittenden ${ }^{b}$, and Gerard \\ Rowe $^{a}$
}

\begin{abstract}
The copper- and iron-containing metal-organic frameworks (MOFs) HKUST-1 and MIL-100(Fe) absorb organic molecules into their pores. When loaded with electron-rich oligothiophenes, these MOFs react under heat to initiate oxidative polymerization of entrapped monomers. This reaction is not observed in the non-redox-active MOF MIL-100(AI). The resulting MOF composites contain conjugated polymer dopants trapped inside their pores, causing profound shifts in the composite electronic structure. We have characterized the composites by infrared, Raman, and UV-visible spectroscopy and examined their structure using optical microscopy, scanning electron microscopy, and atomic force microscopy. Reasoning from TD-DFT calculations of an HKUST-1 model system bound to monomers, we rationalize the observed reactivity and propose an initiation mechanism based on a ligand-to-metal charge transfer state.
\end{abstract}

\section{Introduction}

Polythiophene (PT) and its derivatives may constitute the most well-studied group of conjugated polymers, in close competition with polyanilines. PTs are electron-rich $p$-type semiconductors, and can be readily doped by oxidation to increase their conductivity. ${ }^{1}$ PTs find commercial applications in organic electronics where flexible conductors or semiconductors are desirable, with a well-known commercial application in antistatic coatings ${ }^{2}$ and a number of emerging uses in photovoltaics ${ }^{3}$, electrochemical sensors or indicators ${ }^{4-6}$, and electrochromic devices. ${ }^{7,8}$ One of the best-known uses of PTs is in bulk heterojunction (BHJ) photovoltaic devices, in which a solution containing both a PT and an electron acceptor is deposited (usually by spin coating) onto a substrate. The resulting thin film can exhibit nanoscale phase separation of the PT and electron acceptor, effectively constituting a nanostructured material which can separate excitons into dissociated charge carriers. ${ }^{9-11}$ This nanoscale structure allows PT blends with an electron acceptor (most often fullerenes such as PCBM, with many others ${ }^{12}$ reported) to serve as the core of efficient thin-film solar cells, especially poly(3-alkylthiophene)s. This self-assembling "soft matter" bulk heterojunction architecture has led to a number of highly efficient devices ${ }^{3,13,14}$ but suffers from various degradation processes which place inherent

\footnotetext{
${ }^{a}$ University of South Carolina Aiken, Department of Chemistry and Physics. 471 University Parkway, Aiken, SC, USA. Tel: 1.803.641.3409; E-mail: nicholasm@usca.edu. http://orcid.org/0000-0001-8048-0857 ${ }^{b}$ University of South Carolina, Department of Physics and Astronomy. 712 Main St., Columbia, SC, 28208, USA.

$\dagger$ Electronic Supplementary Information (ESI) available: Additional SEM and AFM images, output files for DFT calculations, and additional EDS and IR spectroscopy of materials. See DOI: 10.1039/b000000x/
}

limits on BHJ cells. Many of the degradation modes of BHJ cells involve alteration of the nanoscale phase separation due to heat cycling or diffusion. ${ }^{15}$ Inspired by the structure of these cells, we sought to design a composite PT material which could have similarly sized conjugated polymer domains, but in which the other phase would be composed of a crystalline lattice rather than the hard-to-control precipitated fullerene domains. This PT-in-lattice material would serve as a starting point in our search for composites with useful properties, potentially replacing BHJ layers in photovoltaics. The synthesis of these lattice materials might also enable the construction of unusually durable organic/inorganic hybrid electrochromic materials or organic light-emitting materials, as PTs are well-known to serve in both those roles. Metalorganic frameworks (MOFs) are a natural choice for this sort of investigation, as it is well known that many MOFs can absorb organic molecules into their large, regular pores. Furthermore, the pores of many MOFs are a few $\mathrm{nm}$ in diameter ${ }^{16}$ and therefore on the order of the desirable domain size for polymers in heterojunctions, i.e., an order of magnitude below the diffusion length of an exciton in a conjugated polymer. ${ }^{14}$ For these reasons, and out of a desire to create an interesting and new material architecture, we sought to make a hybrid material consisting of conjugated polymers integrated into MOFs by a two-step process of filling the MOF pores with monomer followed by initiation of oxidative polymerization.

A few other reports exist of MOFs derivatized by formation of conjugated polymers inside their pores, including PEDOT formed in a $\mathrm{Zn}$-based $\mathrm{MOF}^{17}$ and PT nanorods templated by a coordination polymer. ${ }^{18}$. We were particularly interested to see Jiao's work forming polypyrrole in a Ni/Zn based MOF using only atmospheric oxygen as an oxidant, particularly since Jiao et al. construct devices using their technique which use the conductivity of the polymer, demonstrating good electrochemical contact between PPy and carbon nanotubes. ${ }^{19}$. Haldar et al. have also very recently reported a conjugated polymer/MOF composite in thin film form, ${ }^{20}$ and Qiu et al. used HKUST-1 itself as a template for electropolymerization of aniline. ${ }^{21}$ As we set out to develop our own techniques for MOF/PT hybrid preparation, we stumbled upon an exciting discovery; MOFs of some redox-active metals including copper and iron can induce oxidative polymerization of oligothiophenes innately, without the application of an external oxidant such as ferric chloride. (Figure 1)

This finding recalls an older work of Millar et al. which found that copper-exchanged zeolites could induce oxidative polymerization. ${ }^{22}$ The reaction takes place with equal facility in air or ultrapure nitrogen atmosphere. To our knowledge, the present work is the first example of a MOF/conjugated polymer preparation technique which does not rely upon an external 


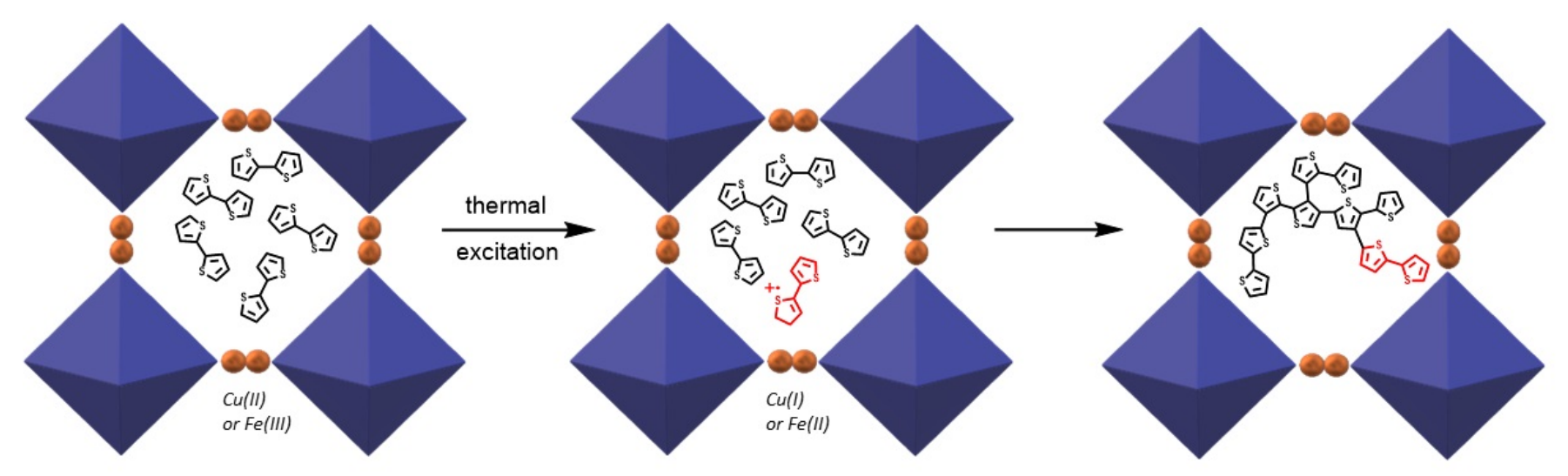

Fig. $1 \mathrm{~A}$ schematic of the proposed for the formation of MOF/polythiophene composite, involving initiation of polymerization by a radical cation produced through a MLCT transition from bound monomer.

oxidant. This discovery provides a convenient and useful route to access $\mathrm{MOF} /$ conjugated polymer hybrids in bulk and thin film form. Furthermore, the discovery that the reaction takes place due to innate oxidation of guest molecules by MOF metal centers must be taken into account in efforts to prepare new MOF hybrids, especially if such initiation would be undesirable.

\section{Experimental}

\section{Computational methods}

All calculations were carried out using Orca 4.0.1.2 for Linux. ${ }^{23}$ GaussView $5.0^{24}$ was used for visualization of orbitals and electron/spin densities. The metal-organic framework was simulated with a simple dicopper paddlewheel complex formed with 4 benzoate groups built in the molecular editor Avogadro 1.2.0. ${ }^{25}$ The appropriate thiophene-based molecule was placed on the $\mathrm{Cu}-\mathrm{Cu}$ bond axis, bound through its sulfur atom. Geometry optimizations were carried out at the PBE/def2-SVP level of theory ${ }^{26,27}$ with Grimme's dispersion correction (D3BJ) ${ }^{28,29}$. To correct for basis set superposition error, all calculations employed the geometrical counterpoise correction $(\mathrm{gCP})^{30}$. For each optimized structure, the assignment of an energetic minimum was confirmed through numerical frequency calculations (i.e., no imaginary frequencies). The electronic spectrum of 1 was predicted with TDA-TD-DFT calculations using the PBE-optimized geometry at the B3LYP/def2-SVP level of theory ${ }^{31}$. Visualization of selected electronic excitations was achieved through the use of TD-DFT difference densities to identify those transitions with thiophene HOMO-based ligand-to-metal charge transfer character.

\section{Spectroscopy and Microscopy}

X-ray photoelectron spectroscopy. XPS measurements were taken at the University of South Carolina's Center for Engineering and Computing, using a Kratos Axis Ultra DLD instrument equipped with a monochromated $\mathrm{Al} \mathrm{K} \alpha$ source and hemispherical analyzer. On indium tin oxide and glass substrates, the carbon $1 \mathrm{~s}$ line was used as a binding energy reference and set to a value of $284.6 \mathrm{eV}$. Fitting of peaks was performed with the SciDAVis software package.

Atomic force microscopy. All AFM measurements were performed on a Dulcinea/Cervantes AFM from Nanotec Electronica using a silicon tip and cantilever operated in a noncontact "tapping" mode with an approximately $70 \mathrm{kHz}$ resonance frequency. The freely available software package WSxM was used for visualization and analysis of images. ${ }^{32}$

Scanning electron microscopy. SEM images were acquired and EDX spectra/element mapping was performed on an Hitachi Cold Field Emission 8200 Series FE-SEM at the Applied Research Center facility, Aiken, SC, USA. A $9 \mathrm{kV}$ accelerating voltage was used for typical images shown.

\section{Infrared spectroscopy.}

Infrared spectra were acquired on a Nicolet 380 spectrometer with a Smart Orbit diamond ATR attachment. 64 scans were summed at a $4 \mathrm{~cm}^{-1}$ resolution to produce a typical spectrum.

\section{Raman spectroscopy and microscopy.}

Raman spectra were acquired on a Renishaw InVia Raman microscope equipped with 4 independent laser lines (488nm, $514 \mathrm{~nm}, 633 \mathrm{~nm}$, and $785 \mathrm{~nm}$ ). After initial testing to maximize signal, the $488 \mathrm{~nm}$ laser was used for all samples shown, with selected spectra repeated using the $785 \mathrm{~nm}$ laser to check for artifacts. Spectra shown are the sum of 5 individual scans.

UV-Vis spectroscopy.

UV-vis spectra of thin films were acquired on a Vernier Instruments single beam VSP-UV instrument with a linear CCD detector and deuterium/incandescent dual sources, reported by the manufacturer to have a wavelength resolution of $2 \mathrm{~nm}$. This instrument was customized in our lab for the purpose of easily accommodating flat solid substrates.

Routine ${ }^{1} \mathrm{H}$ and ${ }^{13} \mathrm{C}$ NMR of monomers were taken using a Anasazi EFT $60 \mathrm{MHz}$ FT-NMR spectrometer referenced to TMS at 0 ppm.

\section{General synthetic remarks}

Unless otherwise indicated, solvents and reagents were obtained from Fisher Scientific and used as received. ITO coated glass 
slides were purchased from NanoCS. Trimesic acid trimethyl ester was prepared from trimesic acid by refluxing a toluene/methanol mixture in an apparatus fitted with a Dean-Stark trap. $18 \mathrm{M} \Omega$ water from a central lab system was used in all applications requiring water. Routine ${ }^{1} \mathrm{H}$ and ${ }^{13} \mathrm{C}$ NMR of monomers were taken using a Anasazi EFT $60 \mathrm{MHz}$ FT-NMR spectrometer referenced to TMS at $0 \mathrm{ppm}$.

\section{Solvothermal MOF syntheses}

HKUST-1 was synthesized according to literature procedures. ${ }^{33}$ Briefly, in a Parr-type autoclave (a PTFE cylinder sealed in a stainless steel threaded cylinder) HKUST-1 was prepared by a twosolvent method. Trimesic acid(0.68g, 3.2mmol) was added to ethanol $(30 \mathrm{~mL})$ in a glass beaker. In a separate container copper(II) nitrate $(2.6 \mathrm{~g}, 10.7 \mathrm{mmol})$ was added to deionized water(30mL). Both mixtures were then poured into the PTFE autoclave cylinder. After stirring the mixture, $\mathrm{DMF}(2 \mathrm{~mL})$ was added, and stirring is continued until solids are dissolved. The autoclave was then sealed and placed into an oven at $80^{\circ} \mathrm{C}$ for 20 hours. The HKUST- 1 was activated by dispersing the MOF into DMF and heating to $80^{\circ} \mathrm{C}$ for 24 hours, followed by filtering on a glass frit and washing with water, ethanol, and chloroform. The activated MOF was dried in a $150^{\circ} \mathrm{C}$ oven for 24 hours. After drying the HKUST-1 was very quickly moved to a desiccator.

MIL-100(Fe) was synthesized according to the procedure of Janiak et al. ${ }^{34}$. Trimesic acid(5.3 mmol) was dissolved in DMSO(40 $\mathrm{mL}$ ) in a 3 neck round bottom flask with stir bar and thermometer. In a separate container, iron(III) nitrate nonahydrate $(3.24 \mathrm{~g}$, $8 \mathrm{mmol}$ ) was dissolved in deionized water $(8 \mathrm{~mL})$. To avoid precipitation of iron(III) nitrate, the iron nitrate solution was heated to $90^{\circ} \mathrm{C}$ before adding it to the trimesic acid solution. The iron (III) nitrate solution was added to the 3-necked flask, and the mixture was heated to $130^{\circ} \mathrm{C}$ for 30 minutes, then cooled to $80^{\circ} \mathrm{C}$ and stirred for 24 hours. The red-orange precipitate of MIL$100(\mathrm{Fe})$ was activated by directly adding the reaction product into DMSO:water (4:1) and stirring for 24 hours. The mixture was centrifuged, the solvent was replaced with ethanol, and the mixture stirred for 24 hours. Finally, the solid was collected by centrifuging and air-dried for 12 hours at $80^{\circ} \mathrm{C}$. After drying in an oven, the MIL-100(Fe) was quickly placed into a desiccator.

MIL-100(Al) was synthesized in bulk by a literature procedure. ${ }^{35}$ Trimesic acid trimethyl ester $(0.20 \mathrm{~g}, 0.79 \mathrm{mmol})$, aluminum nitrate nonahydrate $(0.46 \mathrm{~g}, 1.23 \mathrm{mmol})$ and concentrated nitric acid $(1.54 \mathrm{~mL})$ were added to water $(5.6 \mathrm{~mL})$. This mixture was then added to a Parr-type autoclave and heated to $210^{\circ} \mathrm{C}$ for 4 hours. The solid was collected by centrifugation. MIL-100(Al) was activated by centrifuging the solution and placing the solid into DMF. The MIL-100(Al) and DMF mixture was then heated to $100^{\circ} \mathrm{C}$ for 24 hours. After cooling the MIL-100Al was sequentially washed by centrifugation and replacement of the solvent using ethanol, then chloroform. After air-drying in an oven, the MIL-100(Al) was placed into a descicator.

\section{Preparation of MOF thin films}

Thin films of metal-organic frameworks were made using a modification of the Eddaoudi procedure ${ }^{36}$ by spincasting alternating solutions of the ligand and metal ion on a glass or ITO substrate. Before MOF deposition, the substrate was cleaned by sonicating 10 minutes in ethanol and blown dry in a stream of nitrogen. The ligand solution used was a $3.0 \mathrm{mmol}$ solution of 1,3,5-benzenetricarboxylic acid (trimesic acid). The metal solution used was a $1.5 \mathrm{mmol}$ solution of either anhydrous copper acetate in ethanol for HKUST-1, or ferric nitrate nonahydrate for MIL-100(Fe). Three separate flasks were then filled with the metal solution, the ligand solution and pure ethanol. The spincasting was performed in cycles of metal solution followed by ligand, with a rinsing application of pure ethanol between each solution application. Each application was 50 microliters in volume, applied over 5 seconds of duration for the metal solution and 10 seconds for the ligand solution. Washing with pure ethanol was performed for 10 seconds per washing step. After 50 cycles, the thin film was washed with more ethanol and air-dried in an oven at $100^{\circ} \mathrm{C}$ for 24 hours.

\section{Preparation of bulk MOF composites}

An activated sample of MOF(0.05g) was weighed out and placed into a scintillation vial. Care was taken to do this process quickly to retain dryness. Monomer was added to each vial. For liquid monomers (EDOT and bithiophene) enough liquid was used to cover the surface or solid. $30 \mathrm{mg}$ was used in the case of terthiophene. In sealed vials, each monomer/MOF combination was then reacted at a series of different temperatures, viz., room temperature for $24 \mathrm{~h}, 100^{\circ} \mathrm{C}$ for 1 hour and $100^{\circ} \mathrm{C}$ for $24 \mathrm{~h}$. After reaction, each composite was washed and filtered with water, ethanol and chloroform.

\section{Preparation of HKUST-1 bulk composite, airfree}

HKUST-1(0.05g) was weighed out and placed into a $10 \mathrm{~mL}$ Schlenk fask under ultrapure nitrogen on a Schlenk line. Vacuum (5 Torr) was applied to the flask while heating heated above $100^{\circ} \mathrm{C}$ using a heat gun. The flask was placed back under an ultrapure nitrogen atmosphere. Bithiophene $(2 \mathrm{~mL})$ was added to the flask and the mixture heated with a heat gun. After cooling, the solid was washed and filtered with water, ethanol and chloroform and air-dried in a dessicator.

\section{Preparation of MOF thin film composite}

Thin films of MOF were reacted to form composite in a procedure similar to bulk material. A few drops of neat monomer, sufficient to visibly cover the surface, were placed onto the surface of the film in a sealed vial. The sample in the sealed vial was reacted for the selected time. For instance, bithiophene was placed onto the surface of an HKUST-1 film and was left at room temperature, 1 hour and 24 hours. After the reaction time, the film was washed with water, ethanol, and chloroform. 


\section{Results and Discussion}

We developed our procedure for composite synthesis using the well-known benzenetricarboxylate MOFs HKUST-1 and MIL$100(\mathrm{Fe})$, which incorporate $\mathrm{Cu}(\mathrm{II})$ and $\mathrm{Fe}(\mathrm{III})$ respectively. We found that a solvothermal procedure was suitable for preparing thick, crystalline deposits of HKUST-1 on glass and ITO substrates as well as in the bulk phase. The Eddaoudi group ${ }^{36}$ recently published a convenient alternative procedure for making HKUST1 thin films on glass by spin-casting alternating solutions of the metal salt and linker. We adapted this procedure for the preparation of MIL-100(Fe) and MIL-100(Al) films on ITO as well. Initially, we sought to impregnate oligothiophenes into these MOFs in bulk and thin film form, followed by application of an external oxidant to generate a hybrid material. As summarized in the Introduction, this approach based on an external oxidant has been reported by other groups. However, we noticed during our first experiments that upon heating bithiophene-treated HKUST-1 film, or letting it stand at ambient temperature for approximately one day, a dramatic color change occurred without the addition of any other reactants. The HKUST-1 thin film developed a very deep blue-green color (appearing nearly black) and the edges of the film displayed a red tint characteristic of free polythiophene. We inferred that an oxidative polymerization reaction was occurring under these conditions.

We extended this result to bulk samples of the HKUST-1 and MIL-100(Fe) MOFs, finding that washed and activated samples of these MOFs suspended in liquid bithiophene spontaneously reacted to form a durable green-black composite (Figure 2 over the course of several days. SEM and AFM imaging of HKUST-1 samples before and after treatment with bithiophene and terthiophene (Figures 3 and 4) showed that treated and untreated material had effectively identical morphologies, ruling out mere physisorption of the highly insoluble PT.

In particular, a single face of a large solvothermally prepared HKUST-1 crystal treated with bithiophene is molecularly flat (Figure 5) and indistinguishable from untreated material (Figure S9) rather than showing the characteristic bumpy morphology of PT. The morphology matches literature AFM images of HKUST- $1,{ }^{37}$ even after treatment with 2,2'-bithiophene, although no clear steps corresponding to the reported growth pattern in this material are observed in either of our images. The thin-film samples of HKUST-1 prepared by spin-casting have a similarly robust nanoscale morphology, being indistinguishable to SEM after treatment with bithiophene and terthiophene despite dramatic changes in color. We attempted a similar experiment with the well-known and readily polymerized thiophene monomer EDOT. Unsurprisingly, EDOT reacts readily, yielding dramatic dark-colored hybrid materials both in thin film and bulk form. In this case, however, imaging at the nanoscale shows that the structures are visibly coated with PEDOT. The distinctive triangular facets of HKUST-1 crystals are still apparent, but covered with a rough film consistent with the insoluble, rigid polythiophene PEDOT. (Figure 6)

EDS analysis supports the assignment of the nanostructures

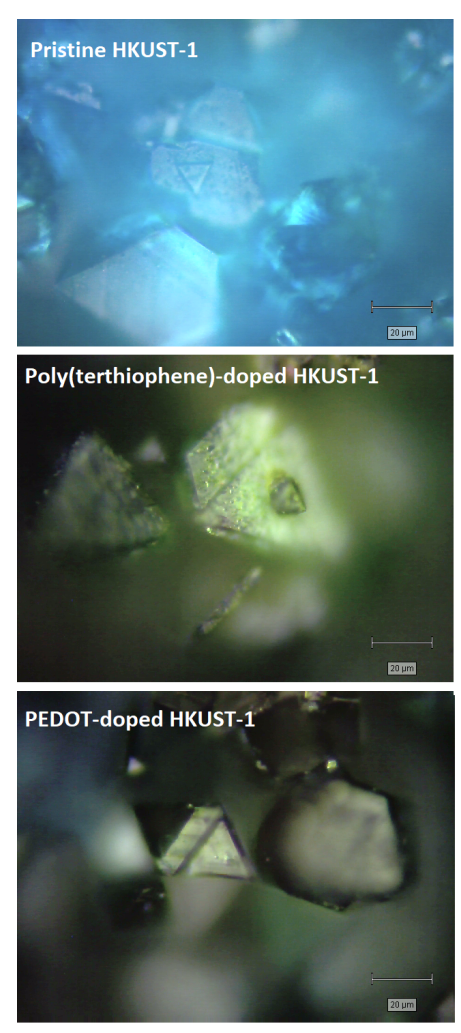

Fig. 2 HKUST-1 shows a dramatic change in color upon heating with oligothiophene and EDOT monomers, without detectable changes in morphology.

from Figure 6 as PEDOT-coated HKUST-1; features of the film are high in oxygen and sulfur relative to background by EDS. (Figure S7) This heavy deposition of PEDOT makes it difficult to easily determine whether the nanostructures are merely coated with polymer, filled with polymer, or some combination of the two possibilities. Based on the UV-vis spectrum of the PEDOT composite, though, (Figure 7) we believe that the HKUST-1/PEDOT material is predominantly the true composite with polymer in the MOF pores, as the same spectral structure (a broadened absorption relative to unmodified HKUST-1 near $400 \mathrm{~nm}$, with a broad tail extending across the entire visible spectrum) is present in the PEDOT composite as in the poly-TT and poly-BT materials. Free PEDOT, with a broader, redder absorption centered at $550 \mathrm{~nm}^{38}$, does not substantially contribute to the spectrum of the PEDOT composite.

Under particularly extreme conditions, heating at $100^{\circ} \mathrm{C}$ for $24 \mathrm{~h}$ in the presence of a large excess of bithiophene, a thin film of HKUST-1 is actually etched by the reaction. This process yields a layer of polythiophene nanostructures still bound to the ITO surface. Traces of the original triangular silhouettes of the MOF surface features can still be seen in the SEM image of the residual polythiophene film after etching. (Figure 11) XPS and IR spectra confirm that the MOF structure is eliminated, as no carboxylate from the trimesate linker remains. However, a substantial amount of copper remains as measured by XPS and EDS. (Figures 10 and S1) We attribute this surprising decomposition of the MOF to reduction of the $\mathrm{Cu}(\mathrm{II})$ centers. $\mathrm{Cu}(\mathrm{I})$ has a relatively small affin- 

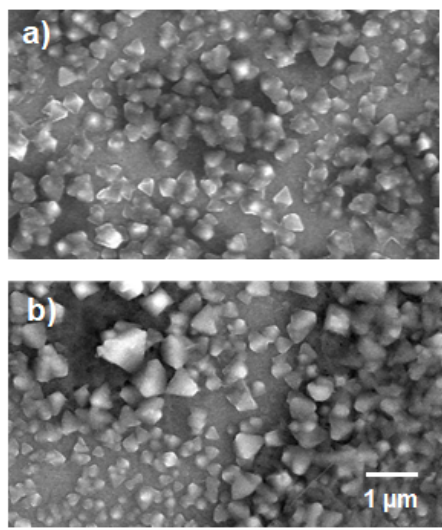

Fig. 3 Morphology of HKUST-1 thin films (prepared in a single batch) remains indistinguishable (a) before and (b) after $1 \mathrm{~h}$ heating with neat bithiophene.

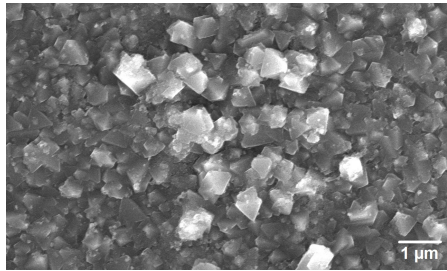

Fig. 4 The terthiophene/HKUST-1 composite, like the bithiophene composite, appears unchanged in SEM imaging from a pristine HKUST-1 thin film despite dramatic differences in vibrational and electronic spectra.

ity for carboxylate ligands, ${ }^{39}$ as is shown by a straightforward ligand-field argument, and a $\mathrm{Cu}(\mathrm{I})$-based HKUST-1 relative would be unstable and likely degrade during the reaction or afterward during the washing process. If a critical fraction of $\mathrm{Cu}$ (II) centers are reduced in the relatively small crystals which comprise the HKUST-1 thin film, it could cause disintegration of the crystal, since the $\mathrm{Cu}(\mathrm{I})$ sites induce strain in the structure. ${ }^{40}$ The copper remaining in the etched film is largely in the $\mathrm{Cu}(\mathrm{I})$ form based on the energy of the Cu LMM peak, (Figure 10) which may be the form produced by the reaction between the MOF and guest molecules or formed due to air oxidation of metallic $\mathrm{Cu}$ produced by the reaction. Since $\mathrm{Cu}(\mathrm{I})$ can disproportionate into $\mathrm{Cu}$ metal and $\mathrm{Cu}(\mathrm{II})$, we cannot make an inference about the form of copper directly produced in this reaction from the data, but the simplest interpretation of the XPS data is that $\mathrm{Cu}(\mathrm{I})$ is produced and remains metastable in the MOF or polymer matrix on the order of days. Traces of this effect are seen in the Cu 2p XPS spectrum of the bulk material, where the $\mathrm{Cu}(\mathrm{I})$ peak is more intense after treatment with bithiophene. (Figure 8) Other than this small but detectable enhancement in the $\mathrm{Cu}(\mathrm{I})$ peak, the XPS spectrum of bithiophene-modified bulk solvothermal HKUST-1 shows little qualitative difference from that of the pre-treatment MOF.

The most dramatic difference by XPS can be seen in the C $1 \mathrm{~s}$ spectrum of unmodified spin-cast HKUST-1 thin film material versus an etched bithiophene-treated spin-cast thin film. (Figure 9)

In the latter, the peak corresponding to carboxylate carbon is completely missing, and the main $\mathrm{Csp}^{2}$ peak shifted, consistent

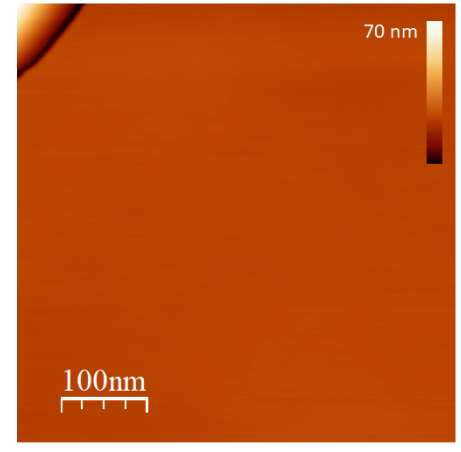

Fig. 5 A single facet of a bithiophene-treated HKUST-1 crystal is flat to AFM, with an RMS roughness of $0.28 \mathrm{~nm}$ and maximum excursion of $2.9 \mathrm{~nm}$ on an individual facet. The edge of the facet is seen at top left.

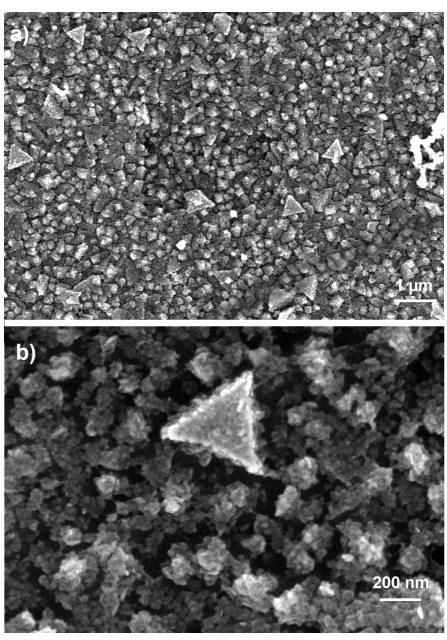

Fig. 6 SEM imaging (a) of a EDOT/HKUST-1 hybrid thin film reveals the distinctive octahedral crystals of HKUST-1 are coated with a rough coating of the conjugated polymer PEDOT, seen particularly clearly in a high-magnification image of a single crystal (b).

with a film comprised primarily of polythiophene in which none of the original MOF survives. This result is surprising considering that remnants of the distinctive triangular structure of HKUST-1 survive in the treated thin film after etching away the MOF, (Figure 11) and these structures are seen by XPS (Figure 10) and EDS imaging (Figure S2) to contain copper. The most straightforward interpretation of these results is that copper ions remain in the polythiophene as a dopant after the destruction of the MOF structure. XPS of the post-etch film indicates $\mathrm{Cu}(\mathrm{I})$ is present, consistent with the $\mathrm{Cu}$ (II) ions in the parent HKUST-1 film acting as the oxidizing agent in the oxidative polymerization of bithiophene.

Soxhlet extraction of a bithiophene-treated HKUST-1 sample (over $72 \mathrm{~h}$, presumably exhaustive) gives a blue-fluorescing supernatant without detectably changing the color or absorption spectrum of the composite solid. Mass spectrometry of this supernatant shows the extracted component to be comprised of oligothiophenes, primarily $\alpha$-quaterthiophene. These oligothiophenes are presumably freed by leaching of the outermost layer of PT-containing HKUST-1; the observed distribution of masses likely overrepresents lighter components due to the greater ease with which smaller oligomers are solubilized and worked free of 


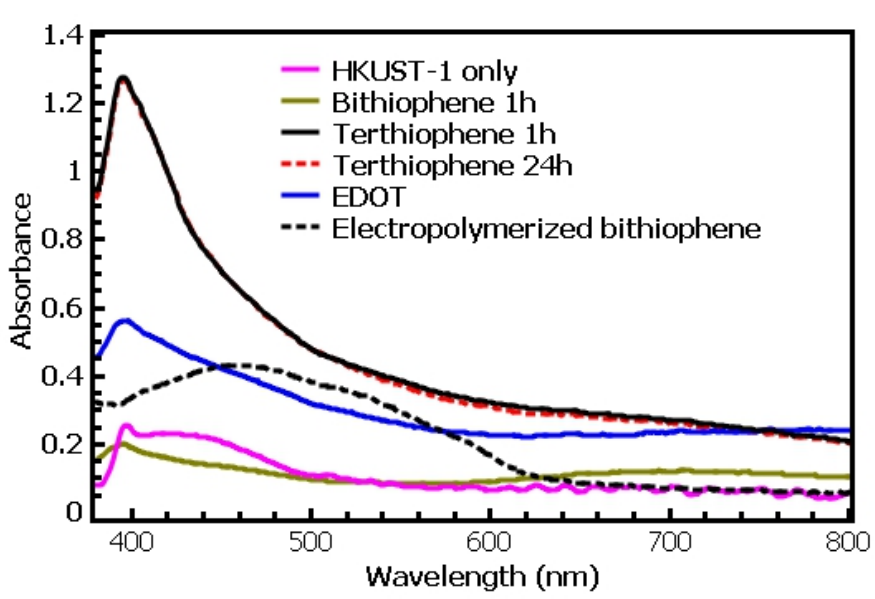

Fig. 7 UV-Vis spectra of spin-cast HKUST-1 thin films treated with oligothiophene monomers, showing the unusual absorption spectrum of the polymer/MOF hybrid. Composite spectra retain the absorption peak near $400 \mathrm{~nm}$, broadened and redshifted.

the HKUST-1 pores.The Raman spectrum of the extracted HKUST$1 /$ bithiophene composite is indistinguishable from that of the asprepared composite.

A SEM survey of the etched HKUST-1/bithiophene composite film turned up an interesting defect which resembles a smiley face. (Figure S2) EDS image mapping of this defect shows a high intensity of silicon, sodium, and aluminum in the exposed background of the "face," and copper and sulfur in the material of the thin film. The clear correlation between the distribution of sulfur and copper, especially in light of the unchanged morphology after bithiophene treatment but before etching (Figure 3 ) is consistent with our hypothesis of an internal polymerization process which localizes polythiophene inside the MOF structure, as well as our identification of the remaining material as copper-doped polythiophene.

Vibrational spectroscopy of the doped bulk MOFs also reveals the presence of the guest polymer. In the IR spectrum, Figure 12 , the $\mathrm{C}-\mathrm{H}$ out-of-plane bend appears at $875 \mathrm{~cm}^{-1}$, a blue-shift of approximately $50 \mathrm{~cm}^{-1}$ from the corresponding mode in bithiophene. ${ }^{41}$ The out-of-plane bend is the most intense peak in many published spectra of oligothiophenes, and is intense in our material. A mode involving the $\mathrm{C}-\mathrm{H}$ in-plane bend can be seen distinctly at $1042 \mathrm{~cm}^{-1}$; conveniently, this peak does not overlap any intense IR peaks in HKUST-1. $\mathrm{C}=\mathrm{C}$ symmetric stretching modes distinguishable from those in the guest MOF can be seen at 1588 and $1640 \mathrm{~cm}^{-1}$ as well. The narrow $\mathrm{C}=\mathrm{C}$ asymmetric stretching mode at $1420 \mathrm{~cm}^{-1}$, seen as a shoulder on the MOF peak at 1446 $\mathrm{cm}^{-1}$, is identified by Sauvajol et al. as due to polythiophene; the related peak at $1470 \mathrm{~cm}^{-1}$, particularly characteristic of the extended conjugation in the polythiophene system, ${ }^{42}$ appears near 1480 in our samples, similar to other reports of oxidatively polymerized 2,2'-bithiophene. ${ }^{43,44}$. The peak at $1047 \mathrm{~cm}^{-1},{ }^{42}$ char-

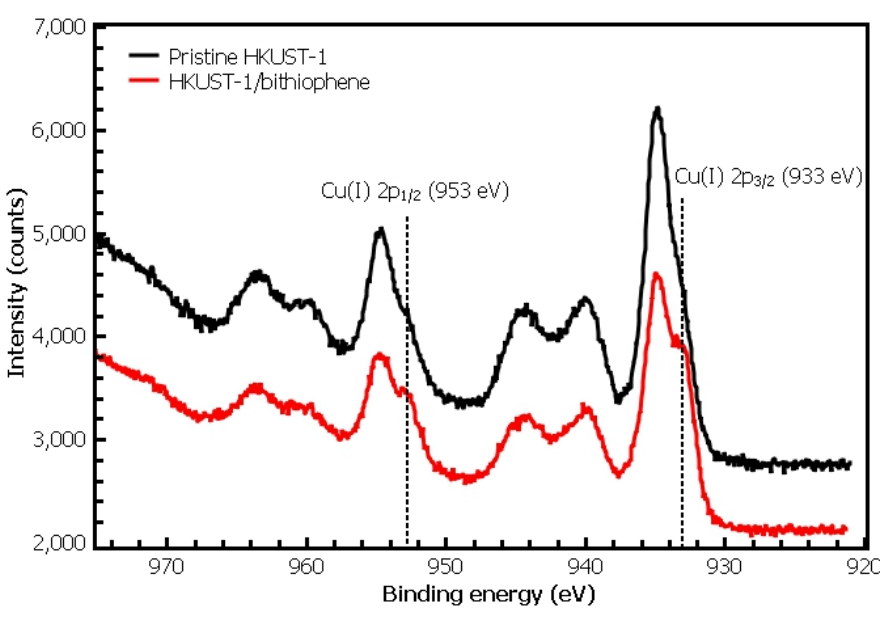

Fig. 8 XPS of the Cu $2 p$ region of bulk HKUST-1 before and after treatment with bithiophene show enhancement of the $\mathrm{Cu}(\mathrm{I})$ peak after reaction.

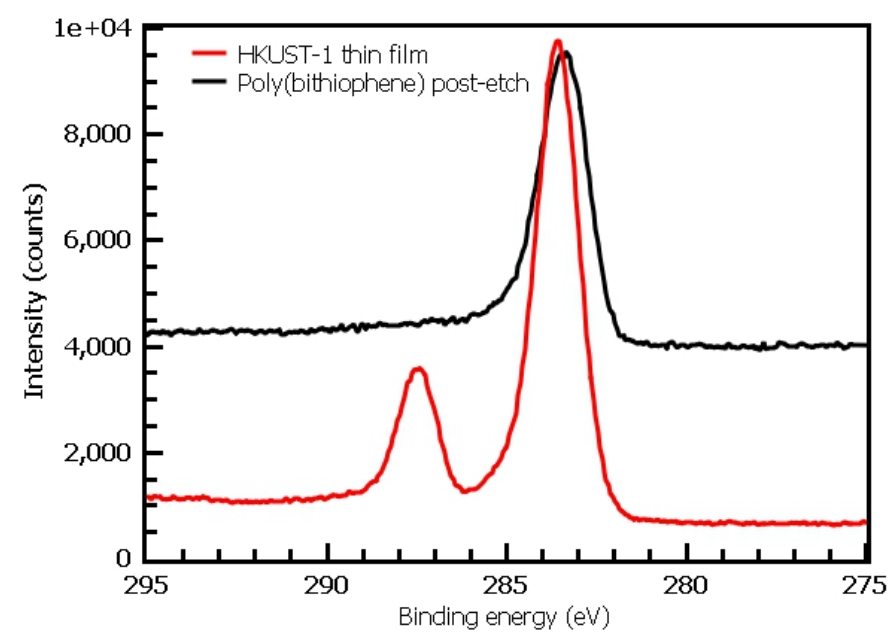

Fig. 9 Etching of a bithiophene-treated HKUST-1 thin film leaves behind a layer of polythiophene nanostructures which show no traces of carboxylate carbon in the $C$ 1s XPS spectrum.

acteristic of polythiophene and conveniently clear of any MOF peaks, is distinctly visible in all doped materials. All Raman spectra of composites are dominated by the spectrum of the conjugated polymer, with the peaks due to the MOF still visible but relatively weak in intensity. This change in the Raman spectrum is quite similar to that observed by Wang et al. ${ }^{17}$ for their PEDOT/MOF composite. The most clearly visible peaks due to polythiophenes are the $\mathrm{C}=\mathrm{C}$ stretching modes centered around 1500 $\mathrm{cm}^{-1}$ and the in-plane $\mathrm{C}-\mathrm{H}$ mode at $1048 \mathrm{~cm}^{-1}$. Many features in the PEDOT composite are similar to those in the polythiophenebased material, save that the Raman spectrum of PEDOT obviously lacks the $\mathrm{C}-\mathrm{H}$ in-plane aromatic modes near $1067 \mathrm{~cm}^{-1}$ and presents a new $\mathrm{C}-\mathrm{O}$ stretch at $1120 \mathrm{~cm}^{-1}$. PEDOT composite also shows unique IR modes at 1189 and $1058 \mathrm{~cm}^{-1}$ corresponding to $\mathrm{C}-\mathrm{O}-\mathrm{C}$ deformations, and a C-S-C deformation at $890 \mathrm{~cm}^{-1}$, higher than that of plain polythiophene. ${ }^{38}$ 

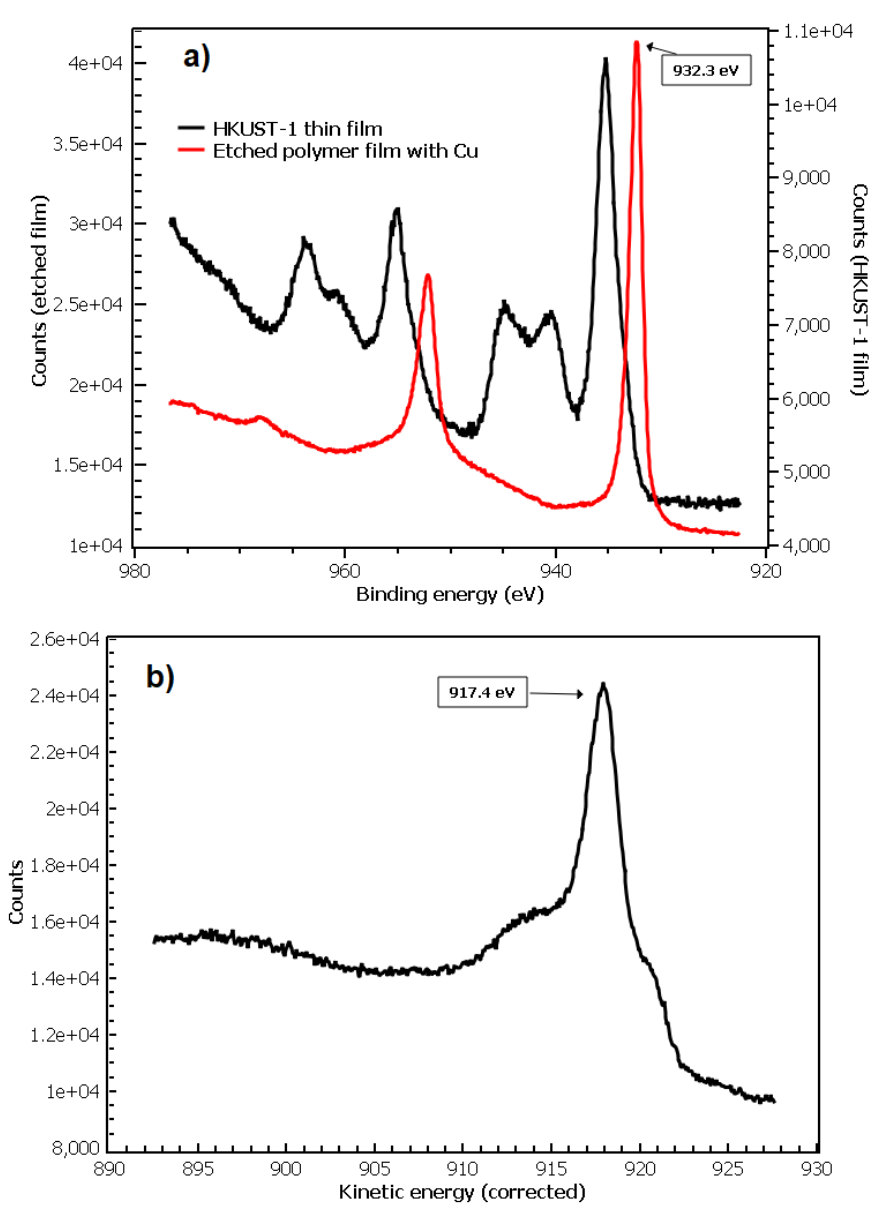

Fig. 10 a) Cu 2p XPS of the bithiophene-treated etched film shows that all remaining copper in the thin film is reduced, while the unmodified HKUST-1 film is entirely in the $\mathrm{Cu}(\mathrm{II})$ state within the limits of detection. b) The Auger parameter for $\mathrm{Cu}$ in the etched film is $1849.7 \mathrm{eV}$, a good match for $\mathrm{Cu}(\mathrm{I})$, calculated from the $\mathrm{Cu} 2 \mathrm{p}_{3 / 2} \mathrm{LMM}$ peak energy difference.

As previously noted, bithiophene and terthiophene composites show no evidence by SEM and AFM of physisorbed polythiophene on the surface; the polymer appears to be contained entirely within the MOF. (Figures 5 and 11) On the other hand, PEDOT is visibly coated on the MOF treated with the monomer EDOT in SEM images. (Figure 6) However, Raman spectroscopy of these three composites are quite comparable in the intensity of the conjugated polymer bands, with the EDOT composite actually being the weakest overall signal of the three samples. While not conclusive, the similarity in vibrational peak intensities between the EDOT-based and thiophene-based materials further bolsters our hypothesis based on the UV-vis spectrum of the EDOT/HKUST hybrid that the EDOT-based material also has pores filled with conjugated polymer. In all Raman spectra, treated materials show peak broadening and a high baseline due to the broad visible and near-IR absorption of the hybrid, which is substantial for all lasers in our Raman microscope. (Figure 7)

Polymer doping has a dramatic effect on the UV-vis spectrum of the material. (Figure 7) We attribute the shift in the primary visible light absorption peak near $400 \mathrm{~nm}$ to a direct interaction

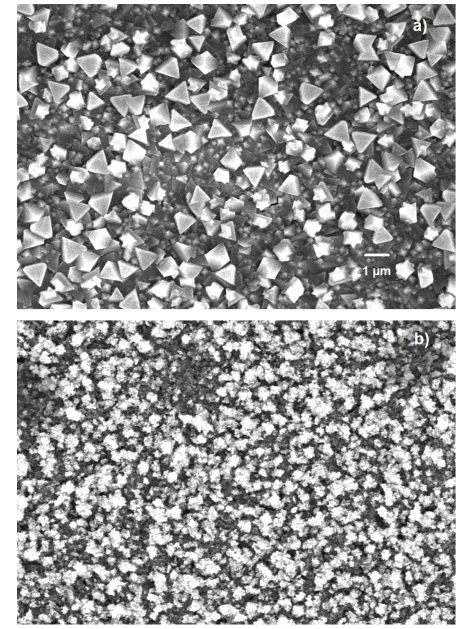

Fig. 11 a) The SEM micrograph of a HKUST-1 thin film treated with bithiophene remains indistinguishable from the pristine MOF. b) After etching, polythiophene nanostructures containing copper remain in the thin film. The scale bar in a) applies to both micrographs.

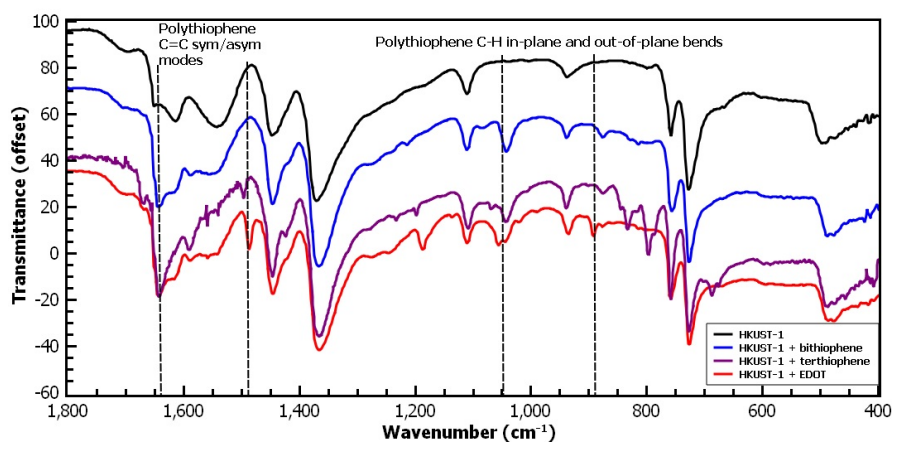

Fig. 12 IR spectroscopy of HKUST-1 before and after treatment with oligothiophenes and EDOT shows the appearance of key peaks corresponding to the conjugated polymer after heating.

of the guest polymer directly to the $\mathrm{Cu}(\mathrm{II})_{2}$ unit in the center of the HKUST-1 paddlewheel structure. This interaction has precedent with small-molecule ligands in HKUST-1, and it is generally known that guest molecules in the HKUST-1 pore complex at the dicopper site 45 and perturb the MOF's absorption spectrum. In the composite, however, a very broad transition appears covering the entire visible region with a blackbody-like "tail". The resulting material absorbs light with an intensity reminiscent of pure mixed copper sulfides. We attribute the broad additional absorption to injection of valence-band electrons from polythiophenes into the conduction band of HKUST-1. Even in unmodified HKUST-1, the lowest-lying excitation is a ligand-metal charge transfer band, occupying a state built from the $\mathrm{Cu} 3 \mathrm{~d}$ orbitals. ${ }^{46}$ This proposed band structure of the composite is consistent with the observed UV-vis spectrum. TD-DFT calculations of complexes between monomers and a HKUST-1 model system (Fig. 14) are also consistent with this interpretation. For instance, an infrared-accessible (ca. $1000 \mathrm{~nm}$ ) excitation moves electron density from both 2,2'bithiophene and terthiophene ligands onto the copper center, ef- 


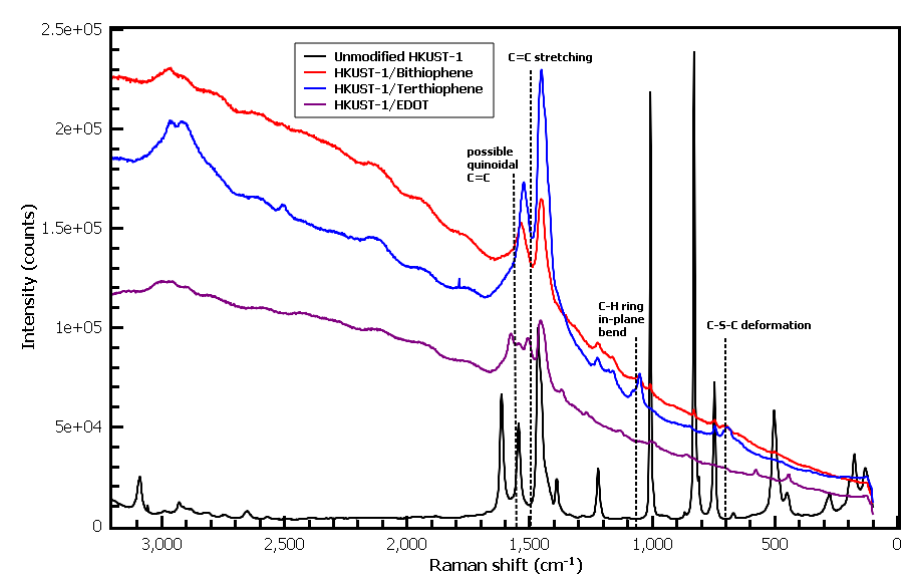

Fig. 13 The Raman spectra of composites is dominated by the polythiophene and PEDOT guest materials, with the distinctive $\mathrm{C}=\mathrm{C}$ stretching doublet clearly visible in all spectra near $1500 \mathrm{~cm}^{-1}$ and the faint but characteristic C-S-C ring deformation appearing at $700 \mathrm{~cm}^{-1}$.

fectively constituting an example of the proposed ligand-to-metal charge transfer band. (Figure 15) This transition also sheds light on the observed (albeit unsurprising) trend in monomer reactivity, in which the heat and time required for reaction decrease in the more electron-rich monomers terthiophene and EDOT relative to bithiophene. In the more electron-rich monomer ligands, the energy of the transition decreases and is more easily accessed thermally.

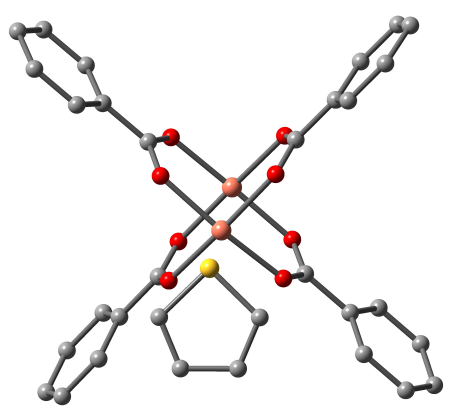

Fig. 14 Our model system for the HKUST-1 consists of a single paddlewheel element built from benzoate ligands on a $\mathrm{Cu}(\mathrm{II})_{2}$ center, complexed to an oligothiophene ligand. (Shown: thiophene)

The excited state produced by the transition effectively constitutes a radical cation centered on the oligothiophene monomer, (Figure 16) a reactive intermediate which will initiate polymerization similarly to well-known processes initiated by oxidizing agents such as persulfates or ferric chloride. ${ }^{20,47}$ The trend in the vertical excitation energies in the present study (decreasing by $0.98 \mathrm{eV}$ from thiophene to bithiophene) show a reasonable similarity to reported vertical ionization potential differences between thiophene and bithiophene performed at a comparable level of theory, which ranged from $1.3 \mathrm{eV}$ in the gas phase to $0.7 \mathrm{eV}$ using a PCM model of acetonitrile. ${ }^{48}$

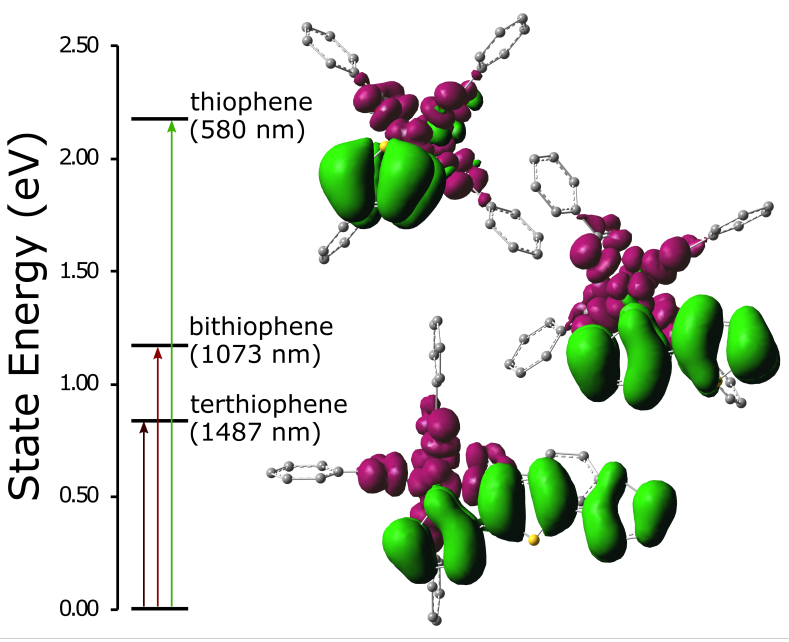

Fig. 15 (Left) Energy diagram showing the TD-DFT determined vertical excitation energies corresponding to charge transfer between oligothiophene HOMO and Cu LUMO with the expected $\mathrm{dx}^{2}-\mathrm{y}^{2}$ character. Energies reported relative to the respective ground state. (Right) Difference density plots for the respective electronic transitions (green = negative electron density change; purple = positive electron density change). We attribute the initiation of polymerization by HKUST-1 to the excited state populated by this transition.

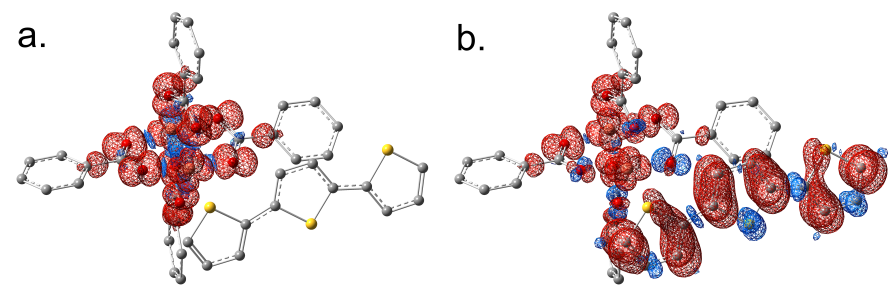

Fig. $16 \mathrm{~A}$ map of unpaired spin density in (a) the triplet ground state and (b) the excited state populated by the low-lying MLCT transition of the oligothiophene/paddlewheel complex shows that the transition confers radical cation character onto the terthiophene ligand, providing an initiator for oxidative polymerization.

In light of $\mathrm{Hu}$ et al's interesting preparation of a MOF/polypyrrole hybrid using oxygen oxidation, and because of a possible role of oxygen in our own system due to potential air oxidation of $\mathrm{Cu}(\mathrm{I})$ centers in the MOF, we tested the effects of excluding oxygen from the HKUST-1 system during the reaction. The HKUST-1/bithiophene composite forms as readily in a wellpurged ultrapure nitrogen environment as it does while open to air, without signs of decomposition of the underlying MOF visible by bright-field optical microscopy. The Raman spectrum of the composite prepared in oxygen-free conditions is essentially indistinguishable from the typical sample prepared in air.

Bright-field microscopy of the composites is well worth examining, not only for the striking appearance of the dark bluegreen doped crystals, (Fig. 2) but for verification on the microscopic scale that the materials, which show clear differences by spectroscopy, are not merely a microscopic blend of unchanged crystals with loose polymer. In our laboratory's experience, optical microscopy often shows features (especially the presence of physisorbed contaminants or extra phases) in materials and 
thin films which are not detectable to the naked eye nor on the nanoscale, but which substantially affect the appearance of the observed spectrum. In the present case, micrographs of the unmodified and modified materials show no obvious amorphous material corresponding to free polymer, but only the distinctive triangular facets of the HKUST-1 MOF. This similarity appears to hold from the microscopic scale down to the nanoscale.

Raman spectroscopy of composites made from MIL-100 bulk solvothermal materials is consistent with the results we predict from HKUST-1 reactivity, with the redox-active Fe(III) based material reacting readily to polymerize oligothiophenes while $\mathrm{Al}(\mathrm{III})$ based MIL-100 shows no traces of polymer. (Fig. 17)
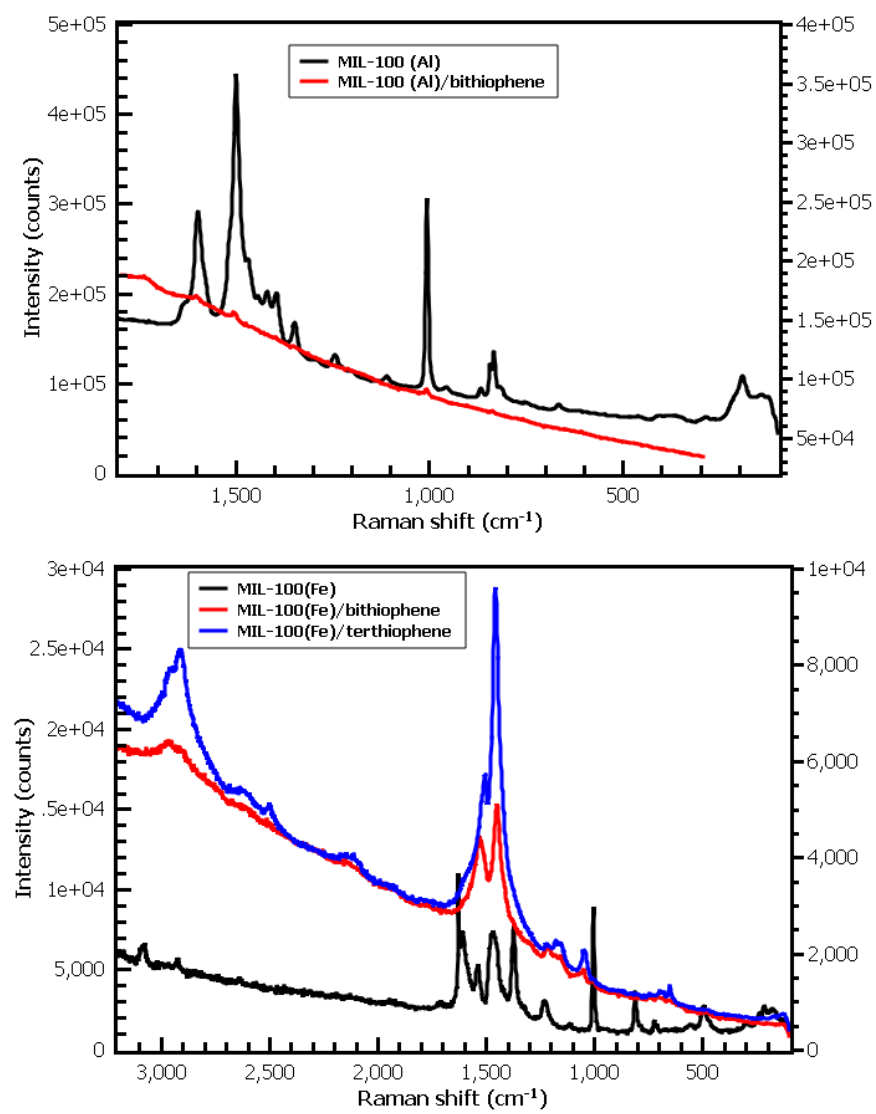

Fig. 17 Raman spectroscopy of solvothermally synthesized MIL-100(Al) at top and MIL-100(Fe) at bottom shows the latter reacting readily to yield polythiophene, while the former does not.

\section{Conclusions}

The redox-active MOFs HKUST-1 and MIL-100(Fe) initiate the oxidative polymerization of thiophene monomers, including bithiophene, terthiophene, and EDOT, absorbed in their pores. The reaction occurs more readily in more electron-rich systems, and unmodified thiophene does not react readily without an external oxidizing agent. Electronic transition energies predicted by TD-DFT calculations to yield radical cation excited states on oligothiophene ligands are good predictors of the observed reactivity series of oligothiophenes. The non-redox-active aluminumbased MOF MIL-100(Al) does not initiate polymerization despite its Lewis acidity. The materials resulting from MOF-initiated polymerization have polythiophenes trapped inside the pores of the MOF. Trapped polymer is detectable by vibrational spectroscopy and causes profound changes in the electronic structure of the material. In a thin film of the MOF produced by spin-casting, the MOF can be etched away, leaving behind a copper-doped polythiophene which still preserves much of the MOF's crystalline nanostructure. Both the doped crystalline MOF and polythiophene nanostructures represent novel techniques in nanomaterial synthesis, and have potential applications in photovoltaics and electrochromic device construction.

\section{Author contributions}

This manuscript version has been approved by all co-authors. NM initiated the project and wrote the manuscript except as noted below. WJ performed all synthetic work, IR, and UV-visible spectroscopy, and wrote the experimental section of the manuscript. JF took and validated all Raman spectra and optical micrographs. SC proposed SPM characterization techniques and took and validated AFM measurements. GR helped propose and devise experiments, performed DFT calculations and analyzed the results, and helped in the interpretation of spectral data.

\section{Acknowledgements}

This project was supported in part by funding provided by the USC Aiken College of Sciences and Engineering Summer Scholars Institute. The Summer Scholars Institute is supported by the Workforce Opportunities in Regional Careers (WORC) grant, ADP, and the South Carolina IDeA Networks of Biomedical Research (SC INBRE) Support was also provided through a RISE grant from the University of South Carolina Office of the Vice President for Research. The authors are particularly grateful to Prof. Chad Leverette (USC Aiken) for assistance in using the InVia Raman microscope, as well as to instrumental experts Mr. Patrick Woodell (Applied Research Center, Aiken, SC, SEM/EDS) and Dr. Stavros Karakalos (USC Columbia) for their skilled work in collecting data on the materials described in this report.

\section{References}

1 Z. G. Soos, Annual Review of Physical Chemistry, 1974, 25, 121-153.

2 K. Reuter, S. Kirchmeyer and A. Elschner, Handbook of Thiophene-Based Materials, Wiley-Blackwell, 2009, pp. 549-576.

3 G. J. Hedley, A. Ruseckas and I. D. W. Samuel, Chemical Reviews, 2017, 117, 796-837.

4 N. Marshall, S. K. Sontag and J. Locklin, Functional Polymer Films, WileyBlackwell, 2011, pp. 319-377.

5 T. P. Kaloni, P. K. Giesbrecht, G. Schreckenbach and M. S. Freund, Chemistry of Materials, 2017, 29, 10248-10283.

6 V. Krikstolaityte, J. Kuliesius, A. Ramanaviciene, L. Mikoliunaite, A. KausaiteMinkstimiene, Y. Oztekin and A. Ramanavicius, Polymer, 2014, 55, 1613-1620.

7 A. L. Dyer, A. M. Österholm, D. E. Shen, K. E. Johnson and J. R. Reynolds, Electrochromic Materials and Devices, Wiley-Blackwell, 2015, pp. 113-184.

8 W. T. Neo, Q. Ye, S.-J. Chua and J. Xu, Journal of Materials Chemistry C, 2016 4, 7364-7376.

9 M. Rita Narayan and J. Singh, Journal of Applied Physics, 2013, 114, 073510.

10 K. B. Whaley, A. A. Kocherzhenko and A. Nitzan, The Journal of Physical Chemistry C, 2014, 118, 27235-27244.

11 W. Chen, T. Xu, F. He, W. Wang, C. Wang, J. Strzalka, Y. Liu, J. Wen, D. J. Miller, J. Chen, K. Hong, L. Yu and S. B. Darling, Nano Letters, 2011, 11, 3707-3713.

12 G. Zhang, J. Zhao, P. C. Y. Chow, K. Jiang, J. Zhang, Z. Zhu, J. Zhang, F. Huang and H. Yan, Chemical Reviews, 2018, 118, 3447-3507.

13 J. Zhang, L. Zhu and Z. Wei, Small Methods, 2017, 1, 1700258. 
14 Y. Tamai, H. Ohkita, H. Benten and S. Ito, The Journal of Physical Chemistry Letters, 2015, 6, 3417-3428.

15 S. Rafique, S. M. Abdullah, K. Sulaiman and M. Iwamoto, Renewable and Sustainable Energy Reviews, 2018, 84, 43-53.

16 Z. Chen, Z.-G. Gu, W.-Q. Fu, F. Wang and J. Zhang, ACS Applied Materials \& Interfaces, 2016, 8, 28737-28742.

17 T. Wang, M. Farajollahi, S. Henke, T. Zhu, S. R. Bajpe, S. Sun, J. S. Barnard, J. S. Lee, J. D. W. Madden, A. K. Cheetham and S. K. Smoukov, Materials Horizons, 2017, 4, 64-71.

18 T. Kitao, M. W. A. MacLean, B. L. Ouay, Y. Sasaki, M. Tsujimoto, S. Kitagawa and T. Uemura, Polymer Chemistry, 2017, 8, 5077-5081.

19 Y. Jiao, G. Chen, D. Chen, J. Pei and Y. Hu, Journal of Materials Chemistry A, 2017, 5, 23744-23752.

20 R. Haldar, B. Sen, S. Hurrle, T. Kitao, R. Sankhla, B. Kühl, A. Welle, S. Heissler, G. Brenner-Weiß, P. Thissen, T. Uemura, H. Gliemann, C. Barner-Kowollik and C. Wöll, European Polymer Journal, 2018, 109, 162-168.

21 C. Lu, T. Ben, S. Xu and S. Qiu, Angewandte Chemie International Edition, 2014, 53, 6454-6458.

22 G. J. Millar, G. F. McCann, C. M. Hobbis, G. A. Bowmaker and R. P. Cooney, Journal of the Chemical Society, Faraday Transactions, 1994, 90, 2579-2584.

23 F. Neese, Wiley Interdisciplinary Reviews: Computational Molecular Science, 2012, 2, 73-78.

24 a. J. M. M. R. Dennington T. A. Kieth, GaussView, 2009.

25 M. D. Hanwell, D. E. Curtis, D. C. Lonie, T. Vandermeersch, E. Zurek and G. R. Hutchison, Journal of Cheminformatics, 2012, 4, 17.

26 F. Weigend and R. Ahlrichs, Physical Chemistry Chemical Physics, 2005, 7, 32973305.

27 J. P. Perdew, K. Burke and M. Ernzerhof, Physical Review Letters, 1996, 77, 38653868.

28 S. Grimme, S. Ehrlich and L. Goerigk, Journal of Computational Chemistry, 2011, 32, 1456-1465.

29 S. Grimme, J. Antony, S. Ehrlich and H. Krieg, The Journal of Chemical Physics, 2010, 132, 154104.

30 H. Kruse and S. Grimme, The Journal of Chemical Physics, 2012, 136, 154101.

31 A. D. Becke, The Journal of Chemical Physics, 1993, 98, 5648-5652.
32 I. Horcas, R. Fernández, J. M. Gómez-Rodríguez, J. Colchero, J. Gómez-Herrero and A. M. Baro, Review of Scientific Instruments, 2007, 78, 013705.

33 S. D. Worrall, M. A. Bissett, W. Hirunpinyopas, M. P. Attfield and R. A. W. Dryfe, Journal of Materials Chemistry C, 2016, 4, 8687-8695.

34 F. Jeremias, S. K. Henninger and C. Janiak, Dalton Transactions, 2016, 45, 86378644.

35 C. Volkringer, D. Popov, T. Loiseau, G. Férey, M. Burghammer, C. Riekel, M. Haouas and F. Taulelle, Chemistry of Materials, 2009, 21, 5695-5697.

36 V. Chernikova, O. Shekhah and M. Eddaoudi, ACS Applied Materials \& Interfaces, 2016, 8, 20459-20464.

37 M. Shöâeè, J. R. Agger, M. W. Anderson and M. P. Attfield, CrystEngComm, 2008, 10, 646-648.

38 M. Wang, R. Jamal, Y. Wang, L. Yang, F. Liu and T. Abdiryim, Nanoscale Research Letters, 2015, 10, 370.

39 Y. Sevryugina, A. Y. Rogachev and M. A. Petrukhina, Inorganic Chemistry, 2007, 46, 7870-7879.

40 M. Todaro, A. Alessi, L. Sciortino, S. Agnello, M. Cannas, F. M. Gelardi and G. Buscarino, Journal of Spectroscopy, 2016, 8074297, 1-7.

41 A. S. Matharu, S. J. Cowling and G. Wright, Liquid Crystals, 2007, 34, 489-506.

42 J. L. Sauvajol, G. Poussigue, C. Benoit, J. P. Lere-Porte and C. Chorro, Synthetic Metals, 1991, 41, 1237-1242.

43 L. Zhu, Y. Niu, Y. Cao, A. Lei, X. Ai and H. Yang, Electrochimica Acta, 2012, 78 27-31.

44 Z. Zhang, F. Wang, F. Chen and G. Shi, Materials Letters, 2006, 60, 1039-1042.

45 C. Prestipino, L. Regli, J. G. Vitillo, F. Bonino, A. Damin, C. Lamberti, A. Zecchina, P. L. Solari, K. O. Kongshaug and S. Bordiga, Chemistry of Materials, 2006, 18, 1337-1346.

46 Z.-G. Gu, L. Heinke, C. Wöll, T. Neumann, W. Wenzel, Q. Li, K. Fink, O. D. Gordan and D. R. T. Zahn, Applied Physics Letters, 2015, 107, 183301.

47 V. M. Niemi, P. Knuuttila, J. E. Österholm and J. Korvola, Polymer, 1992, 33 1559-1562.

48 M. B. Camarada, P. Jaque, F. R. Díaz and M. A. d. Valle, Journal of Polymer Science Part B: Polymer Physics, 2011, 49, 1723-1733. 